\title{
Exact-Approximate Boundary Reachability of Thermoelastic Plates Under Variable Thermal Coupling
}

\author{
George Avalos* \\ Irena Lasiecka ${ }^{\dagger}$
}

September 30, 1999

\begin{abstract}
In this paper, we consider controllability properties of a thermoelastic plate equation, in which the (coupling) coefficient of thermal expansion $\alpha$ is allowed to vary with the properties of the plate. Boundary control is exerted through the free boundary conditions of the plate equation, and through the Robin boundary condition of the temperature. These controls have the physical interpretation, respectively, of inserted forces and moments, and prescribed temperature, all of which act on the edges of the plate. The main result here is that this boundary controlled partial differential equation has the following exact-approximate controllability property: With initial data of finite energy, one can find boundary controls such that the mechanical (plate) variable can be controlled exactly, and the thermal variable approximately. The proof of this result relies on an inverse-type estimate which reconstructs the initial energy for the plate from measurements on the boundary.
\end{abstract}

\section{Introduction}

Let $\Omega$ be a bounded open subset of $\mathbb{R}^{2}$, with smooth boundary $\Gamma$. Furthermore, $\Gamma_{*}$ will be any open and nonempty subset of $\Gamma$. We shall consider here controllability properties of the following system of thermoelasticity:

$$
\left\{\begin{array}{l}
\left\{\begin{array}{l}
\omega_{t t}-\gamma \Delta \omega_{t t}+\Delta^{2} \omega+\operatorname{div}[\alpha(x, y) \nabla \theta]=0 \\
\theta_{t}-\Delta \theta+\theta-\operatorname{div}\left[\alpha(x, y) \nabla \omega_{t}\right]=0
\end{array} \text { on }(0, T) \times \Omega ;\right. \\
\left\{\begin{array}{l}
\Delta \omega+(1-\mu) B_{1} \omega+\alpha \theta=u_{1} \\
\frac{\partial \Delta \omega}{\partial \nu}+(1-\mu) \frac{\partial B_{2} \omega}{\partial \tau}-\gamma \frac{\partial \omega_{t t}}{\partial \nu}+\alpha \frac{\partial \theta}{\partial \nu}=u_{2}
\end{array} \quad \text { on }(0, T) \times \Gamma ;\right. \\
\frac{\partial \theta}{\partial \nu}+\lambda \theta=\left\{\begin{array}{l}
u_{3} \text { on }(0, T) \times \Gamma_{*}, \\
0 \text { on }(0, T) \times \Gamma \backslash \Gamma_{*}
\end{array} ; \quad \lambda \geq 0\right. \\
\omega(t=0)=\omega_{0}, \omega_{t}(t=0)=\omega_{1}, \theta(t=0)=\theta_{0} \text { on } \Omega
\end{array}\right.
$$

Here $\gamma>0$ is a parameter accounting for rotational forces, and is assumed to be small. In addition, $\alpha(x, y) \in C^{2}(\bar{\Omega})$ is a thermal parameter which varies with the properties of the plate

\footnotetext{
${ }^{*}$ Department of Mathematics and Statistics, Texas Tech University, Lubbock, Texas 79409. The research of G. Avalos is partially supported by the NSF Grant DMS-9710981.

†'Department of Mathematics, Thornton Hall, University of Virginia, Charlottesville, Va 22903. The research of I. Lasiecka is partially supported by the NSF Grant DMS-9804822, and by the Army Research Office Grant DAAH0498-1-0059.
} 
material. Moreover, $\mu \in(0,1)$ is Poisson's ratio. The (natural) boundary operators $B_{i}$ are defined as

$$
\begin{aligned}
& B_{1} \equiv 2 \nu_{1} \nu_{2} \frac{\partial^{2}}{\partial x \partial y}-\nu_{1}^{2} \frac{\partial^{2}}{\partial y^{2}}-\nu_{2}^{2} \frac{\partial^{2}}{\partial x^{2}} \\
& B_{2} \equiv\left(\nu_{1}^{2}-\nu_{2}^{2}\right) \frac{\partial^{2}}{\partial x \partial y}+\nu_{1} \nu_{1}\left(\frac{\partial^{2}}{\partial y^{2}}-\frac{\partial^{2}}{\partial x^{2}}\right) .
\end{aligned}
$$

There are other physical parameters associated with this model, but without loss of generality, they have been set here to unity. The boundary functions $u_{i}$ are taken to be the "controls" of this partial differential equation (PDE) system, and are each $L^{2}$ in time into a (to be) specified Hilbert space. In the absence of these boundary controls, one has wellposedness in the finite energy space

$$
\mathbf{H}_{\gamma} \equiv H^{2}(\Omega) \times H^{1}(\Omega) \times L^{2}(\Omega) .
$$

That is to say, with $u_{i}=0, i=1,2,3$; and initial data $\left[\omega_{0}, \omega_{1}, \theta_{0}\right] \in \mathbf{H}_{\gamma}$, one has that the corresponding solution to the $\operatorname{PDE}(1)$ satisfies $\left[\omega, \omega_{t}, \theta\right] \in C\left([0, T] ; \mathbf{H}_{\gamma}\right)$. Here, we wish to determine reachability properties of the solution $\left[\omega, \omega_{t}, \theta\right]$, corresponding to controls $u_{i}$ of certain smoothness. Our main result is the following:

Theorem 1.1 Let $T>2 \sqrt{\gamma} \operatorname{diam}(\Omega)$. Then the following controllability property holds true: for given initial data $\left[\omega_{0}, \omega_{1}, \theta_{0}\right]$ and terminal data $\left[\omega_{0}^{T}, \omega_{1}^{T}, \theta_{0}^{T}\right]$ in the space $\mathbf{H}_{\gamma}$, and arbitrary $\epsilon>0$, one can find control functions $\left[u_{1}^{*}, u_{2}^{*}, u_{3}^{*}\right] \in L^{2}\left(0, T ; L^{2}(\Gamma)\right) \times L^{2}\left(0, T ; H^{-1}(\Gamma)\right) \times C^{\infty}\left(\Sigma_{*}\right)$ such that the corresponding solution $\left[\omega^{*}, \omega_{t}^{*}, \theta^{*}\right]$ to (1) satisfies at terminal time $T$,

$$
\begin{aligned}
{\left[\omega^{*}(T), \omega_{t}^{*}(T)\right] } & =\left[\omega_{0}^{T}, \omega_{1}^{T}\right] ; \\
\left\|\theta^{*}(T)-\theta_{0}^{T}\right\|_{L^{2}(\Omega)} & \leq \epsilon .
\end{aligned}
$$

This theorem is essentially a corollary from the following result of controllability for the mechanical variable only:

Theorem 1.2 Let $T>2 \sqrt{\gamma} \operatorname{diam}(\Omega)$. Then for all initial data $\left[\omega_{0}, \omega_{1}, \theta_{0}\right] \in \mathbf{H}_{\gamma}$, and terminal data $\left[\omega_{0}, \omega_{1}\right] \in H^{2}(\Omega) \times H^{1}(\Omega)$, there exists $\left[u_{1}, u_{2}, u_{3}\right] \in L^{2}\left(0, T ; L^{2}(\Gamma)\right) \times L^{2}\left(0, T ; H^{-1}(\Gamma)\right) \times H^{s}\left(\Sigma_{*}\right)$, where arbitrary $s \geq 0$, such that the corresponding solution $\left[\omega, \omega_{t}, \theta\right]$ to (1) satisfies $\left[\omega(T), \omega_{t}(T)\right]=$ $\left[\omega_{0}^{T}, \omega_{1}^{T}\right]$.

Once the partial exact controllability result Theorem 1.2 is established, one can easily invoke the constructive procedure outlined in [3] to find the control $\left[u_{1}^{*}, u_{2}^{*}, u_{3}^{*}\right]$ which meets the demands of Theorem 1.1. Accordingly, we concentrate below on the proof of Theorem 1.2 only.

Remark 1.3 The results of Theorems 1.1, 1.2 also hold for the case when the mechanical controls $\left[u_{1}, u_{2}\right]$ are applied on $\Gamma_{1} \subset \Gamma$, where $\Gamma_{0}=\Gamma \backslash \Gamma_{1}$ satisfies suitable geometric conditions and $\overline{\Gamma_{0}} \cap \overline{\Gamma_{1}}=$ $\varnothing($ see $[3])$.

\section{Literature Related to the Problem}

Questions related to approximate-exact controllability of thermoelastic systems have attracted considerable attention in recent years. The simplest case to consider in this context is that of internal controllability, where the problem reduces to addressing the exact controllability of the mechanical variable (wave or plate), the solution of which being now well-known. Indeed, in this case, one can show that the associated controllability operator for the thermoelastic plate can be written as 
a compact perturbation of the controllability operator corresponding to the (uncoupled) Kirchoff plate only. And for the latter problem, the surjectivity of the corresponding controllability map is well-established (see [17],[26] and references therein). This fact, together with the approximate controllability of the original thermoelastic system, and the available literature concerning the theory of compact perturbations of controllable systems (see e.g., [29],[4],[11]), allows the deduction of approximate-exact controllability for the PDE (1).

The situation is very different in the case of boundary control problems, which usually cannot be viewed as mere compact perturbations of the "mechanical" part of the equation. This is particularly so for the case of control via free edge boundary conditions. In this case, in addition to an unavailable decomposition of the thermoelastic semigroup into a mechanical plate semigroup and a compact perturbation, there is also the added complication that the associated boundary control operator is unbounded on the basic state space (see [23]). This last feature is due to the failure of the Lopatinski condition to be satisfied for the thermoelastic plate with free boundary conditions. In view of these complications, boundary controllability results for systems of thermoelasticity are much harder to obtain, and new ideas must necessarily come into play.

In fact, the very first results in this area are, predictably, for one-dimensional models (see [10]), where the optimal solution to the exact controllability problem was obtained by solving an appropriate moment problem-a very successful one-dimensional tool.

In the case of two-dimensional problems, partial exact controllability (with respect to the mechanical variable) for free edge boundary conditions was first obtained in [27]. The main technique employed there was a multiplier method with differential multipliers. The result obtained in [27] called for a restriction on the size of the coupling (constant) parameter $\alpha$. Most recently, [28] has derived a similar controllability result with a similar restriction on the size of the thermal coupling, for thermoelastic waves. This assumption that the coupling between the thermal and mechanical components be sufficiently "small" may very well be plausible physically, and in agreement with the physical principles underlying the derivation of the model; however, from a mathematical point of view, one could hardly disagree that the removal of this smallness assumption is highly desirable. If not for mathematical elegance and completeness, then certainly a robust controllability theory calls for the removal of unnecessary restrictions involving the size of the physical parameters. Indeed, in the construction of control laws for the controlled PDE (1), a dependency on small parameters in the model may lead to very unpleasant and unpredictable effects at the level of implementation.

This was precisely a motivation behind [3], where the exact-approximate boundary controllability of the PDE (1), with $\alpha$ constant, was obtained without restricting the size of the thermal coupling. This was achieved by: (i) introducing elements of microlocal analysis into the multiplier estimates developed in [27]; (ii) applying special TDO multipliers for the 2-D system of thermoelasticity, these multipliers making their initial appearance in [2] (in the context of uniform stability) ; (iii) resorting to a new unique continuation property for the system of thermoelasticity in [16]. The results obtained in [2] not only dispense with size constraints placed on the coupling coefficients, but also do away with the need for assuming geometric conditions (star-shaped in particular) which have appeared customary in previously posted results on the boundary controllability of waves and plates (see [17]).

All the results reported above deal with the case of constant coefficients. This paper, instead, is the first to tackle the controllability of thermoelastic plates with variable coefficients. It is wellknown and recognized in the literature that the case of variable coefficients, in the context of controllability, is an altogether different problem. The main obstacle is the fact that standard differential multipliers are no longer effective on problems where variations of the coefficients occur at the energy and higher level. In this case, Carleman's estimates appear necessary, even in the case of a simple wave equation. Thus, the tools become more complicated and more technical.

In order to cope with the issue, we shall use very special $H^{-1} \times L^{2}$ recovery estimates, obtained for the perturbed wave equation with variable coefficients. (Analogous estimates were obtained in 
[13]. See also [14] and [15].) These estimates will then be applied to an appropriately rescaled controllability map corresponding to the thermoelastic problem. Recent unique continuation results in [16] and [6] for variable coefficients (see also [5]), together with the new backwards uniqueness property established in [25] are critical in completing the argument.

\section{Proof of Theorem 1.2}

Remark 3.1 Note that the controllability of the system (1) is equivalent to the controllability of $\left(1^{*}\right)$, where $\left(1^{*}\right)$ is the same thermoelastic PDE as that present in (1), but equipped instead with the mechanical boundary conditions

$$
\left\{\begin{array}{l}
\Delta \omega+(1-\mu) B_{1} \omega+\alpha \theta=u_{1} \\
\frac{\partial \Delta \omega}{\partial \nu}+(1-\mu) \frac{\partial B_{2} \omega}{\partial \tau}-\gamma \frac{\partial \omega_{t t}}{\partial \nu}+\alpha \frac{\partial \theta}{\partial \nu}-\omega=u_{2}
\end{array} \text { on }(0, T) \times \Gamma .\right.
$$

Indeed, it suffices to redefine $u_{2}^{*}=u_{2}+\left.\omega\right|_{\Gamma}$, in order to obtain the controllability of the original system (1). This last assertion follows from the fact that the map $\left.\left[u_{1}, u_{2}, u_{3}\right] \rightarrow \omega\right|_{\Gamma}$ is a bounded mapping of $L^{2}(\Sigma) \times L^{2}\left(0, T ; H^{-1}(\Gamma)\right) \times C^{\infty}(\Sigma)$ into $C\left([0, T] ; H^{\frac{1}{2}}(\Gamma)\right) \subset L^{2}(\Sigma)$.

Given the Remark 3.1, our proof of partial exact controllability (again, with respect to the mechanical variable) will center on analyzing the solution $\left[\phi, \phi_{t}, \psi\right] \in C\left([0, T] ; \mathbf{H}_{\gamma}\right)$ of the "adjoint" $\mathrm{PDE}$ system (adjoint with respect to the $\mathrm{PDE}\left(1^{*}\right)$ ),

$$
\left\{\begin{array}{l}
\left\{\begin{array}{l}
\phi_{t t}-\gamma \Delta \phi_{t t}+\Delta^{2} \phi+\operatorname{div}[\alpha(x, y) \nabla \psi]=0 \\
\psi_{t}+\Delta \psi-\psi-\operatorname{div}\left[\alpha(x, y) \nabla \phi_{t}\right]=0
\end{array} \text { on } Q\right. \\
\left\{\begin{array}{l}
\Delta \phi+(1-\mu) B_{1} \phi+\alpha \psi=0 \\
\frac{\partial \Delta \phi}{\partial \nu}+(1-\mu) \frac{\partial B_{2} \phi}{\partial \tau}-\gamma \frac{\partial \phi_{t t}}{\partial \nu}+\alpha \frac{\partial \psi}{\partial \nu}-\phi=0
\end{array} \quad \text { on } \Sigma\right. \\
\frac{\partial \psi}{\partial \nu}+\lambda \psi=0 \text { on } \Sigma, \quad \lambda \geq 0 \\
{\left[\phi(T), \phi_{t}(T), \psi(T)\right]=\left[\phi_{0}, \phi_{1}, 0\right]}
\end{array}\right.
$$

(note the terminal thermal condition $\psi(T)=0$ reflects the fact that we are out to exactly the mechanical variable only). With this solutions of this PDE in mind, the energy of the mechanical variable at time $t$ will be denoted as $E_{\phi}(t)$, this being defined as

$$
E_{\phi}(t) \equiv a(\phi(t), \phi(t))+\left\|\phi_{t}(t)\right\|_{L^{2}(\Omega)}^{2}+\gamma\left\|\nabla \phi_{t}(t)\right\|_{L^{2}(\Omega)}^{2},
$$

where the bilinear form $a(\cdot, \cdot)$ is defined by

$$
a(\phi, \widehat{\phi}) \equiv \int_{\Omega}\left[\phi_{x x} \widehat{\phi}_{x x}+\phi_{y y} \widehat{\phi}_{y y}+\mu\left(\phi_{x x} \widehat{\phi}_{y y}+\phi_{y y} \widehat{\phi}_{x x}\right)+2(1-\mu) \phi_{x y} \widehat{\phi}_{x y}\right] d \Omega+\int_{\Gamma} \phi \widehat{\phi} d \Gamma .
$$

(In particular then, $\left.E_{\phi}(T)=a\left(\phi_{0}, \phi_{0}\right)+\gamma\left\|\nabla \phi_{1}\right\|_{L^{2}(\Omega)}^{2}+\left\|\phi_{1}\right\|_{L^{2}(\Omega)}^{2}\right)$. It is known that $C_{1}\left\|\phi_{0}\right\|_{H^{2}(\Omega)} \leq$ $\sqrt{a\left(\phi_{0}, \phi_{0}\right)} \leq C_{2}\left\|\phi_{0}\right\|_{H^{2}(\Omega)}$ for all $\phi_{0} \in H^{2}(\Omega)$, so that $\sqrt{a(\cdot, \cdot)} \sim\|\cdot\|_{H^{2}(\Omega)}$. In what follows below, we will have occasion to appeal to the following Green's Theorem for the biharmonic operator (see [26]): For two functions $\phi, \widehat{\phi}$ of sufficient smoothness, there is the relation

$$
\begin{aligned}
& \int_{\Omega}\left(\Delta^{2} \phi\right) \widehat{\phi} d \Omega+\int_{\Gamma} \phi \widehat{\phi} d \Gamma \\
= & a(\phi, \widehat{\phi})+\int_{\Gamma}\left[\frac{\partial \Delta \phi}{\partial \nu}+(1-\mu) \frac{\partial B_{2} \phi}{\partial \nu}\right] \widehat{\phi} d \Gamma-\int_{\Gamma}\left[\Delta \phi+(1-\mu) B_{1} \phi\right] \frac{\partial \widehat{\phi}}{\partial \nu} d \Gamma .
\end{aligned}
$$


Now for $s$ real, we define $\mathcal{U}_{s}$ to be the product of spaces

$$
\mathcal{U}_{s} \equiv L^{2}\left(0, T ; L^{2}(\Gamma)\right) \times L^{2}\left(0, T ; H^{-1}(\Gamma)\right) \times H^{s}\left(\Sigma_{*}\right) .
$$

With this space, we denote $\mathcal{L}_{T}: D\left(\mathcal{L}_{T}\right) \subset \mathcal{U}_{s} \rightarrow \mathbf{H}_{\gamma}$ to be the "control to terminal state" map; that is, for all $\left[u_{1}, u_{2}, u_{3}\right] \in D\left(\mathcal{L}_{T}\right)$,

$$
\mathcal{L}_{T}\left[\begin{array}{l}
u_{1} \\
u_{2} \\
u_{3}
\end{array}\right]=\left[\begin{array}{c}
\omega(T) \\
\omega_{t}(T) \\
\theta(T)
\end{array}\right],
$$

where $\left[\omega, \omega_{t}, \theta\right]$ is the solution to $(1)$, corresponding to control triple $\left[u_{1}, u_{2}, u_{3}\right]$. Note that $\mathcal{L}_{T}$ is an unbounded operator, owing to the particular mechanical boundary conditions being imposed. (Note however, that the mapping $\left[u_{1}, u_{2}\right] \rightarrow\left[\omega, \omega_{t}, \theta\right]$ is as an element of

$$
\mathcal{L}\left(L^{2}\left(0, T ; L^{2}(\Gamma) \times H^{-1}(\Gamma)\right), C\left([0, T] ; H^{\frac{3}{2}}(\Omega) \times H^{\frac{1}{2}}(\Omega) \times L^{2}(\Omega)\right) ; \text { see [24]), Moreover, let } \Pi \in\right.
$$
$\mathcal{L}\left(\mathbf{H}_{\gamma}, H^{2}(\Omega) \times H^{1}(\Omega)\right)$ denote the following projection:

$$
\Pi\left[\begin{array}{l}
\omega_{0} \\
\omega_{1} \\
\theta_{0}
\end{array}\right]=\left[\begin{array}{l}
\omega_{0} \\
\omega_{1}
\end{array}\right] .
$$

It is readily seen that establishing the partial exact controllability of the system $\left(1^{*}\right)$ is equivalent to showing the surjectivity of the map $\Pi \mathcal{L}_{T}: \mathcal{D}\left(\mathcal{L}_{T}\right) \subset \mathcal{U}_{s} \rightarrow \mathbf{H}_{\gamma}$. By the classical functional analysis then (see e.g., [12], Lemma 3.8.18, p. 102), the proof of Theorem 1.2 will be complete if we can ascertain the existence of a constant $C_{T}>0$ (again for $T>\sqrt{\gamma} \operatorname{diam}(\Omega)$ ) such that for all $\left[\phi_{0}, \phi_{1}\right] \in \mathcal{D}\left(\mathcal{L}_{T}^{*} \Pi^{*}\right) \subset H^{2}(\Omega) \times H^{1}(\Omega)$, one has the following inverse inequality

$$
\int_{0}^{T}\left\|\nabla \phi_{t}\right\|_{L^{2}(\Gamma)}^{2} d t+\|\psi\|_{\left[H^{s}\left(\Sigma_{*}\right)\right]^{\prime}}^{2} \geq C_{T} E_{\phi}(T),
$$

where $\left.\phi_{t}\right|_{\Gamma}$ and $\left.\psi\right|_{\Gamma_{*}}$ are traces of solution $\left[\phi, \phi_{t}, \psi\right]$ to the backwards system (4). Our work below is geared towards establishing the inequality $(7)$.

Before starting, we first note that in regards to the PDE (4), one can show by a standard energy argument (see e.g., [27], [2]) that for arbitrary terminal data $\left[\phi_{0}, \phi_{1}\right]$, the thermal component of the solution $\left[\phi, \phi_{t}, \psi\right]$ satisfies the estimate

$$
\int_{0}^{T}\|\nabla \psi\|_{L^{2}(\Omega)}^{2} d t \leq C E_{\phi}(T)
$$

In view of this additional regularity, we denote as "l.o.t. $\left(\phi, \phi_{t}, \psi\right)$ " (lower order terms), all quantities which are below level of energy; that is, for every $\epsilon>0$,

$$
\begin{aligned}
\text { I.o.t. }\left(\phi, \phi_{t}, \psi\right) \leq & C_{T}\left(\|\phi\|_{L^{\infty}\left(0, T ; H^{2-\epsilon}(\Omega)\right)}+\left\|\phi_{t}\right\|_{L^{\infty}\left(0, T ; H^{1-\epsilon}(\Omega)\right)}+\|\psi\|_{L^{\infty}\left(0, T ; H^{-\epsilon}(\Omega)\right)}\right. \\
& \left.+\int_{0}^{T}\|\psi\|_{H^{1-\epsilon}(\Omega)}^{2} d t\right) .
\end{aligned}
$$

In addition, we shall use the standard denotation $\mathcal{O}(\cdot)$, where

$$
a=\mathcal{O}(b) \Leftrightarrow|a| \leq C b \text { for some constant } C .
$$

Finally, by invoking a density argument, we can throughout assume solutions $\left[\phi, \phi_{t}, \psi\right]$ to (4), corresponding to smooth terminal data, which have the regularity required to justify the computations to be done below.

We first need the following estimate on the mechanical energy. 
Lemma 3.2 Let $T>2 \sqrt{\gamma} \operatorname{diam}(\Omega)$. Then for all $0<\epsilon_{0}<\frac{T}{2}$, we have the estimate

$$
\int_{\epsilon_{0}}^{T-\epsilon_{0}} E_{\phi}(t) d t \leq C_{T, \epsilon_{0}} \int_{0}^{T}\left\|\nabla \phi_{t}\right\|_{L^{2}\left(\Gamma_{1}\right)}^{2} d t+\text { l.o.t. }\left(\phi, \phi_{t}, \psi\right) .
$$

Proof of Lemma 3.2: Multiplying the backwards heat equation of (4) by $\alpha(x, y)$, and adding the resulting expression to the Kirchoff plate of (4), we obtain the single equation

$$
\phi_{t t}-\gamma \Delta \phi_{t t}+\Delta^{2} \phi=\alpha \psi_{t}-\alpha \nabla \alpha \cdot \nabla \phi_{t}-\alpha^{2} \Delta \phi_{t}-\nabla \alpha \cdot \nabla \psi-\alpha \psi .
$$

Making the change of variable $z \equiv \Delta \phi$, we then have that $z$ satisfies the wave equation

$$
\gamma z_{t t}=\Delta z+\alpha^{2} z_{t}+F
$$

where

$$
F \equiv \frac{d}{d t} f_{1}+f_{2}, \text { with } f_{1}=\phi_{t}-\alpha \psi+\alpha \nabla \alpha \cdot \nabla \phi \text { and } f_{2}=\nabla \alpha \cdot \nabla \psi+\alpha \psi .
$$

Appealing to the recovery estimate in the Appendix for wave equations with forcing term of the form in (12) (see Lemma 4.2 of the Appendix), we have for $T>2 \sqrt{\gamma} \operatorname{diam}(\Omega)$

$$
\begin{aligned}
\int_{0}^{T} & {\left[\|z(t)\|_{L^{2}(\Omega)}^{2}+\left\|z_{t}(t)\right\|_{H^{-1}(\Omega)}^{2}\right] d t } \\
\leq C_{T} & \left(\int_{0}^{T}\|z\|_{L^{2}(\Gamma)} d t+\left\|\frac{\partial z}{\partial \nu}\right\|_{H^{-1}(\Sigma)}^{2}+\|z\|_{H^{-1}(Q)}^{2}\right. \\
& \left.+\int_{0}^{T}\left[\left\|f_{1}\right\|_{L^{2}(\Omega)}^{2}+\left\|f_{2}\right\|_{H^{-1}(\Omega)}^{2}\right] d t+\left\|f_{1}\right\|_{C\left([0, T] ; H^{-\frac{1}{2}+\epsilon}(\Omega)\right)}^{2}\right) .
\end{aligned}
$$

Transforming back to the mechanical variable, this estimate becomes

$$
\begin{aligned}
& \int_{0}^{T}\left[\|\Delta \phi(t)\|_{L^{2}(\Omega)}^{2}+\left\|\Delta \phi_{t}(t)\right\|_{H^{-1}(\Omega)}^{2}\right] d t \\
\leq & C_{T}\left(\int_{0}^{T}\|\Delta \phi\|_{L^{2}(\Gamma)}^{2} d t+\left\|\frac{\partial \Delta \phi}{\partial \nu}\right\|_{H^{-1}(\Sigma)}^{2}+\|\Delta \phi\|_{H^{-1}(Q)}^{2}\right. \\
& \left.+\int_{0}^{T}\left[\left\|f_{1}\right\|_{L^{2}(\Omega)}^{2}+\left\|f_{2}\right\|_{H^{-1}(\Omega)}^{2}\right] d t+\left\|f_{1}\right\|_{C\left([0, T] ; H^{-\frac{1}{2}+\epsilon}(\Omega)\right)}^{2}\right) .
\end{aligned}
$$

We estimate this right hand side. First off, we note from the free boundary conditions in (4) that

$$
\frac{\partial \Delta \phi}{\partial \nu}=(\mu-1) \frac{\partial}{\partial \tau} \frac{\partial^{2} \phi}{\partial \nu \partial \tau}+\gamma \frac{\partial \phi_{t t}}{\partial \nu}+\lambda \alpha \psi+\phi
$$

in consequence, we have

$$
\begin{aligned}
& \left\|\frac{\partial \Delta \phi}{\partial \nu}\right\|_{H^{-1}(\Sigma)} \leq C\left[\left\|\frac{\partial}{\partial \tau} \frac{\partial^{2} \phi}{\partial \nu \partial \tau}\right\|_{H^{-1}(\Sigma)}+\left\|\frac{\partial \phi_{t t}}{\partial \nu}\right\|_{H^{-1}(\Sigma)}+\|\lambda \alpha \psi\|_{H^{-1}(\Sigma)}+\|\phi\|_{H^{-1}(\Sigma)}\right] \\
\leq & C_{\mu, \alpha} \int_{0}^{T}\left[\left\|\frac{\partial^{2} \phi}{\partial \nu \partial \tau}\right\|_{L^{2}(\Gamma)}^{2}+\left\|\frac{\partial \phi_{t}}{\partial \nu}\right\|_{L^{2}(\Gamma)}^{2}+\|\psi\|_{L^{2}(\Gamma)}^{2}+\|\phi\|_{L^{2}(\Gamma)}^{2}\right] d t .
\end{aligned}
$$


We thus have from $(14),(15)$ and a simple rewriting of $\left.\Delta \phi\right|_{\Gamma}$ that

$$
\begin{aligned}
& \int_{0}^{T}\left[\|\Delta \phi(t)\|_{L^{2}(\Omega)}^{2}+\left\|\Delta \phi_{t}(t)\right\|_{H^{-1}(\Omega)}^{2}\right] d t \\
\leq & C_{T}\left(\int_{0}^{T}\left[\left\|\nabla \phi_{t}\right\|_{L^{2}(\Gamma)}^{2}+\left\|\frac{\partial^{2} \phi}{\partial \tau^{2}}\right\|_{L^{2}(\Gamma)}^{2}+\left\|\frac{\partial^{2} \phi}{\partial \nu \partial \tau}\right\|_{L^{2}(\Gamma)}^{2}+\left\|\frac{\partial^{2} \phi}{\partial \nu^{2}}\right\|_{L^{2}(\Gamma)}^{2}\right] d t\right. \\
& \left.+\int_{0}^{T}\left[\left\|f_{1}\right\|_{L^{2}(\Omega)}^{2}+\left\|f_{2}\right\|_{H^{-1}(\Omega)}^{2}\right] d t+\left\|f_{1}\right\|_{C\left(0, T ; H^{-\frac{1}{2}+\epsilon}(\Omega)\right)}^{2}+\text { l.o.t }\left(\phi, \phi_{t}, \psi\right)\right) .
\end{aligned}
$$

Note that the term on the left hand side above does not determine the energy of $\phi(t)$; (i.e., $\left.\|\Delta \phi(t)\|_{L^{2}(\Omega)}^{2}+\left\|\Delta \phi_{t}(t)\right\|_{H^{-1}(\Omega)}^{2} \nsim E_{\phi}(t)\right)$. However, we can use the estimate (16) to majorize $E_{\phi}(t)$.

Indeed, by standard elliptic theory,

$$
\begin{aligned}
\|\phi\|_{H^{2}(\Omega)} & \leq C\left(\|\Delta \phi\|_{L^{2}(\Omega)}+\left\|\gamma_{0} \phi\right\|_{H^{\frac{3}{2}}(\Gamma)}\right) \\
\left\|\phi_{t}\right\|_{H^{1}(\Omega)} & \leq C\left(\left\|\Delta \phi_{t}\right\|_{H^{-1}(\Omega)}+\left\|\gamma_{0} \phi_{t}\right\|_{H^{\frac{1}{2}}(\Gamma)}\right) .
\end{aligned}
$$

This thus gives

$$
\begin{aligned}
& \int_{0}^{T}\left[\|\phi\|_{H^{2}(\Omega)}^{2}+\left\|\phi_{t}\right\|_{H^{1}(\Omega)}^{2}\right] d t \\
\leq & C \int_{0}^{T}\left[\left\|\nabla \phi_{t}\right\|_{L^{2}(\Gamma)}^{2}+\|\Delta \phi\|_{L^{2}(\Omega)}^{2}+\left\|\Delta \phi_{t}\right\|_{H^{-1}(\Omega)}^{2}+\left\|\frac{\partial^{2} \phi}{\partial \tau^{2}}\right\|_{L^{2}(\Gamma)}^{2}\right] d t .
\end{aligned}
$$

In turn, this estimate, combined with that in (16), yields

$$
\begin{aligned}
& \int_{0}^{T} E_{\phi}(t) d t \leq \\
& C_{T}\left\{\int_{0}^{T}\left[\left\|\nabla \phi_{t}\right\|_{L^{2}(\Gamma)}^{2}+\left\|\frac{\partial^{2} \phi}{\partial \tau^{2}}\right\|_{L^{2}(\Gamma)}^{2}+\left\|\frac{\partial^{2} \phi}{\partial \nu \partial \tau}\right\|_{L^{2}(\Gamma)}^{2}+\left\|\frac{\partial^{2} \phi}{\partial \nu^{2}}\right\|_{L^{2}(\Gamma)}^{2}\right] d t\right. \\
& \left.\quad+\int_{0}^{T}\left[\left\|f_{1}\right\|_{L^{2}(\Omega)}^{2}+\left\|f_{2}\right\|_{H^{-1}(\Omega)}^{2}\right] d t+\left\|f_{1}\right\|_{C\left([0, T] ; H^{-\frac{1}{2}+\epsilon}(\Omega)\right)}^{2}+\text { I.o.t. }\left(\phi, \phi_{t}, \psi\right)\right\}
\end{aligned}
$$

(recall the definition of the energy $E_{\phi}(t)$ in $(5)$ ). Note that we can perform the same computations as above, to obtain the estimate (19) on the time interval $\left(T-\epsilon_{0}, \epsilon_{0}\right)$. Doing exactly this and subsequently majorizing the norms of second order boundary derivatives of $\phi$, by means of the trace result Lemma 4.1 of the Appendix, as applied to $\phi$ as the solution of the Kirchoff equation in (4), we obtain

$$
\begin{aligned}
& \int_{\epsilon_{0}}^{T-\epsilon_{0}} E_{\phi}(t) d t \leq C_{T}\left\{\int_{0}^{T}\left[\left\|\nabla \phi_{t}\right\|_{L^{2}(\Gamma)}^{2}+\|\operatorname{div}[\alpha \nabla \psi]\|_{\left[H^{\frac{3}{2}-\epsilon}(\Omega)\right]^{\prime}}^{2}\right] d t\right. \\
& +\int_{0}^{T}\left\|f_{2}\right\|_{H^{-1}(\Omega)}^{2} d t+\text { l.o.t. }\left(\phi, \phi_{t}, \psi\right),
\end{aligned}
$$

(where for this estimate we have implicitly used the definition of $f_{1}$ in (12)). It is evidently necessary to deal with the norms in (20) involving $\operatorname{div}[\alpha \nabla \psi]$ and $f_{2}$ : 
(i) To handle $\operatorname{div}[\alpha \nabla \psi]$, we first define $A_{N}: L^{2}(\Omega) \rightarrow L^{2}(\Omega)$ as $A_{N}=-\Delta+I, D\left(A_{N}\right)=$ $\left\{\vartheta \in H^{2}(\Omega)\right.$ such that $\left.\frac{\partial \vartheta}{\partial \nu}=0\right\}$. As for the case of $A_{D}, A_{N}$ is positive definite, self adjoint, with its fractional powers therefore being well-defined. In particular, from [9], we have that

$$
\begin{aligned}
& D\left(A_{N}^{\xi}\right)=H^{2 \xi}(\Omega) \text { for } 0 \leq \xi<\frac{3}{4} \\
& (\nabla \vartheta, \nabla \widetilde{\vartheta})_{L^{2}(\Omega)}+(\vartheta, \widetilde{\vartheta})_{L^{2}(\Omega)}=\left(A_{N}^{\frac{1}{2}} \vartheta, A_{N}^{\frac{1}{2}} \widetilde{\vartheta}\right)_{L^{2}(\Omega)} \quad \text { for } \vartheta, \widetilde{\vartheta} \in H^{1}(\Omega) .
\end{aligned}
$$

With this operator, we then have pointwise in time for every $\varpi \in H^{\frac{3}{2}-\epsilon}(\Omega)$,

$$
\begin{aligned}
& (\operatorname{div}[\alpha(x, y) \nabla \psi], \varpi)_{L^{2}(\Omega)}=-(\alpha \nabla \psi, \nabla \varpi)_{L^{2}(\Omega)}-\lambda(\alpha \psi, \varpi)_{L^{2}(\Gamma)} \\
= & -(\nabla \psi, \nabla(\alpha \varpi))_{L^{2}(\Omega)}-\left(\frac{\partial \psi}{\partial x},\left[\alpha, \frac{\partial}{\partial x}\right] \varpi\right)_{L^{2}(\Omega)} \\
& -\left(\frac{\partial \psi}{\partial y},\left[\alpha, \frac{\partial}{\partial y}\right] \varpi\right)_{L^{2}(\Omega)}-\lambda(\alpha \psi, \varpi)_{L^{2}(\Gamma)} \\
= & -\left(A_{N}^{\frac{1}{4}+\frac{\epsilon}{2}} \psi, A_{N}^{\frac{3}{4}-\frac{\epsilon}{2}}(\alpha \varpi)\right)_{L^{2}(\Omega)}+(\psi, \alpha \varpi)_{L^{2}(\Omega)}-\left(\frac{\partial \psi}{\partial x},\left[\alpha, \frac{\partial}{\partial x}\right] \varpi\right)_{L^{2}(\Omega)} \\
& -\left(\frac{\partial \psi}{\partial y},\left[\alpha, \frac{\partial}{\partial y}\right] \varpi\right)_{L^{2}(\Omega)}-\lambda(\alpha \psi, \varpi)_{L^{2}(\Gamma)},
\end{aligned}
$$

after using (21) and the definition of the commutator $[A, B]=A B-B A$. An application of the Riesz representation theorem thus yields

$$
\left.\int_{0}^{T}\|\operatorname{div}[\alpha(x, y) \nabla \psi]\|_{\left[H^{\frac{3}{2}-\epsilon}(\Omega)\right]^{\prime}}^{2} d t \leq C \int_{0}^{T} \| \psi\right] \|_{H^{\frac{1}{2}+\epsilon}(\Omega)}^{2} d t .
$$

(ii) In estimating the norms involving $f_{2}$ on the right hand side of (20), we note from the definition (12) that the troublesome term is $\nabla \alpha \cdot \nabla \psi$. But for every $\vartheta \in L^{2}(\Omega)$ and $\varpi \in H_{0}^{1}(\Omega)$, we have

$$
(\nabla \alpha \cdot \nabla \vartheta, \varpi)_{L^{2}(\Omega)}=-(\vartheta, \operatorname{div}[\varpi \nabla \alpha])_{L^{2}(\Omega)} .
$$

Consequently, we have that $\nabla \alpha \cdot \nabla(\cdot) \in \mathcal{L}\left(L^{2}(\Omega), H^{-1}(\Omega)\right)^{1}$, and so we deduce that

$$
\int_{0}^{T}\left\|f_{2}\right\|_{H^{-1}(\Omega)}^{2} d t \leq C \int_{0}^{T}\|\psi\|_{L^{2}(\Omega)}^{2} d t .
$$

Combining (20), (22) and (23) gives now the desired estimate (9).

Lemma 3.3 (i) For all $s, \tau \in[0, T]$, and $\epsilon>0$, the mechanical energy of the PDE system (4) satisfies the following relation:

$$
\begin{aligned}
& {\left[E_{\phi}(t)\right]_{t=s}^{t=\tau} } \\
= & \mathcal{O}\left(C_{\epsilon} \int_{0}^{T}\left\|\nabla \phi_{t}\right\|_{L^{2}(\Gamma)}^{2} d t+\epsilon\left\{E_{\phi}(\tau)+E_{\phi}(s)+\int_{0}^{T}\left[E_{\phi}(t)+\|\nabla \psi(t)\|_{L^{2}(\Omega)}^{2}\right] d t\right\}\right) \\
+ & C_{\epsilon} \text { l.o.t. }\left(\phi, \phi_{t}, \psi\right)
\end{aligned}
$$

\footnotetext{
${ }^{1}$ Here is where we require that $\alpha(x, y) \in C^{2}(\bar{\Omega})$.
} 
(ii) For all $s$ and $\tau$, the mechanical energy $E_{\phi}$ obeys the estimate

$$
\begin{aligned}
& E_{\phi}(\tau) \leq C\left[E_{\phi}(s)+C_{\epsilon} \int_{0}^{T}\left\|\nabla \phi_{t}\right\|_{L^{2}(\Gamma)}^{2} d t\right] \\
+ & \epsilon\left\{\int_{0}^{T}\left[E_{\phi}(t)+\|\nabla \psi(t)\|_{L^{2}(\Omega)}^{2}\right] d t\right\}+C_{\epsilon} \text { l.o.t. }\left(\phi, \phi_{t}, \psi\right) .
\end{aligned}
$$

Proof of Lemma 3.3:

(i) We multiply the Kirchoff plate equation (10) by $\phi_{t}$ and integrate in time and space, invoke the Green's theorem in (6) so as to have

$$
\begin{aligned}
& \left.\frac{1}{2} E_{\phi}(t)\right|_{t=\tau} ^{t=s}=\int_{\tau}^{s}\left(\alpha \psi_{t}, \phi_{t}\right) d t-\int_{\tau}^{s}\left(\alpha \psi, \frac{\partial \phi_{t}}{\partial \nu}+\lambda \phi_{t}\right)_{L^{2}(\Gamma)} d t \\
& -\int_{\tau}^{s}\left(\nabla \alpha \cdot \nabla \psi+\alpha \psi+\alpha \nabla \alpha \cdot \nabla \phi_{t}+\alpha^{2} \Delta \phi_{t}, \phi_{t}\right)_{L^{2}(\Omega)} d t .
\end{aligned}
$$

By use of the inequality $a b \leq \epsilon a^{2}+C_{\epsilon} b^{2}$, the trace theorem of Sobolev, and Green's formula we obtain

$$
\begin{aligned}
& \left.\frac{1}{2} E_{\phi}(t)\right|_{t=\tau} ^{t=s}=\int_{\tau}^{s}\left(\alpha \psi_{t}, \phi_{t}\right)_{L^{2}(\Omega)} d t+\int_{\tau}^{s} \int_{\Omega} \alpha^{2}\left|\nabla \phi_{t}\right|^{2} d \Omega d t \\
& +\mathcal{O}\left(\int_{0}^{T}\left\|\nabla \phi_{t}\right\|_{L^{2}(\Gamma)}^{2} d t+\epsilon \int_{0}^{T}\left[\left\|\nabla \phi_{t}\right\|_{L^{2}(\Omega)}^{2}+\|\nabla \psi\|_{L^{2}(\Omega)}^{2}\right] d t\right)+\text { l.o.t. }\left(\phi, \phi_{t}, \psi\right) .
\end{aligned}
$$

(ii) The critical term in (27) which needs to be eliminated is $\int_{\tau}^{s}\left(\alpha \psi_{t}, \phi_{t}\right)_{L^{2}(\Omega)} d t$. To this end, we introduce an operator theoretic multiplier invoked in earlier work (see [2],[3]), for which purpose, we need the following elliptic operators:

(i.e) The operator $A_{D}: L^{2}(\Omega) \rightarrow L^{2}(\Omega)$ is defined by

$$
A_{D}=-\Delta, D\left(A_{D}\right)=H^{2}(\Omega) \cap H_{0}^{1}(\Omega) .
$$

By the characterization in [9], we have $D\left(A_{D}^{\frac{1}{2}}\right)=H_{0}^{1}(\Omega)$.

(ii.e) We define the (Dirichlet) map $D$, by

$$
D g=h \Leftrightarrow \Delta h=0 \text { in } \Omega \text { and }\left.h\right|_{\Gamma}=g .
$$

By elliptic theory, we know that

$$
D \in \mathcal{L}\left(H^{s}(\Gamma), H^{s+\frac{1}{2}}(\Omega)\right) \text { for all real } s .
$$

Now using the inverse of the elliptic operator defined in (28), we apply the multiplier $A_{D}^{-1}\left(\frac{\alpha}{\gamma} \psi\right)$ to the equation (10) and integrate in time and space to obtain

$$
\begin{aligned}
& 0=\int_{\tau}^{s}\left(\phi_{t t}-\gamma \Delta \phi_{t t}, A_{D}^{-1}\left(\frac{\alpha}{\gamma} \psi\right)\right)_{L^{2}(\Omega)} d t \\
& +\int_{\tau}^{s}\left(\Delta^{2} \phi-\alpha \psi_{t}+\alpha \operatorname{div}\left[\alpha \nabla \phi_{t}\right]+\nabla \alpha \cdot \nabla \psi+\alpha \psi, A_{D}^{-1}\left(\frac{\alpha}{\gamma} \psi\right)\right)_{L^{2}(\Omega)} d t .
\end{aligned}
$$


We now rewrite the right hand side of (31), to which purpose we use the heat equation in (4). First off,

$$
\begin{aligned}
& \int_{\tau}^{s}\left(\phi_{t t}, A_{D}^{-1}\left(\frac{\alpha}{\gamma} \psi\right)\right)_{L^{2}(\Omega)} d t \\
= & {\left[\left(\phi_{t}, A_{D}^{-1}\left(\frac{\alpha}{\gamma} \psi\right)\right)_{L^{2}(\Omega)}\right]_{t=\tau}^{t=s}+\int_{\tau}^{s}\left(\phi_{t}, A_{D}^{-1}\left(\frac{\alpha}{\gamma}\left[\Delta \psi-\psi-\operatorname{div}\left(\alpha \nabla \phi_{t}\right)\right]\right)\right)_{L^{2}(\Omega)} d t . }
\end{aligned}
$$

From this and the fact that $A_{D}^{-1} \in \mathcal{L}\left(H^{-1-\epsilon}(\Omega), H_{0}^{1-\epsilon}(\Omega)\right)$, for $\epsilon<\frac{1}{2}$, we obtain

$$
\int_{\tau}^{s}\left(\phi_{t t}, A_{D}^{-1}\left(\frac{\alpha}{\gamma} \psi\right)\right)_{L^{2}(\Omega)} d t=\text { l.o.t. }\left(\phi, \phi_{t}, \psi\right) \text {. }
$$

Secondly, we have

$$
\begin{aligned}
& -\int_{\tau}^{s}\left(\Delta \phi_{t t}, A_{D}^{-1}(\alpha \psi)\right)_{L^{2}(\Omega)} d t=\int_{\tau}^{s}\left(\nabla \phi_{t t}, \nabla A_{D}^{-1}(\alpha \psi)\right)_{L^{2}(\Omega)} d t \\
= & {\left[\left(\nabla \phi_{t}, \nabla A_{D}^{-1}(\alpha \psi)\right)_{L^{2}(\Omega)}\right]_{\tau}^{s}-\int_{\tau}^{s}\left(\phi_{t}, A_{D} A_{D}^{-1}\left(\alpha \psi_{t}\right)\right)_{L^{2}(\Omega)} d t } \\
& -\int_{\tau}^{s}\left(\phi_{t}, \frac{\partial A_{D}^{-1}\left(\alpha \psi_{t}\right)}{\partial \nu}\right)_{L^{2}(\Gamma)} d t .
\end{aligned}
$$

Using again the backwards heat equation in (4), and the identities

$$
\begin{aligned}
\alpha \Delta \psi & =\Delta(\alpha \psi)+[\Delta, \alpha] \psi=-A_{D}\left[\alpha \psi-D\left(\left.\alpha \psi\right|_{\Gamma}\right)\right]+[\Delta, \alpha] \psi ; \\
\alpha \operatorname{div}\left[\alpha \nabla \phi_{t}\right] & =\alpha^{2} \Delta \phi_{t}+\alpha \nabla \alpha \cdot \nabla \phi_{t}=\Delta \alpha^{2} \phi_{t}+\left[\alpha^{2}, \Delta\right] \phi_{t}+\alpha \nabla \alpha \cdot \nabla \phi_{t} \\
& =-A_{D}\left[\alpha^{2} \phi_{t}-D\left(\left.\alpha^{2} \phi_{t}\right|_{\Gamma}\right)\right]+\left[\alpha^{2}, \Delta\right] \phi_{t}+\alpha \nabla \alpha \cdot \nabla \phi_{t} ;
\end{aligned}
$$

we rewrite the last term on the right hand side of (33) as

$$
\begin{aligned}
& \int_{\tau}^{s}\left(\phi_{t}, \frac{\partial A_{D}^{-1}\left(\alpha \psi_{t}\right)}{\partial \nu}\right)_{L^{2}(\Gamma)} d t \\
= & \int_{\tau}^{s}\left(\phi_{t}, \frac{\partial}{\partial \nu}\left[D \gamma_{0}\left(\alpha^{2} \phi_{t}-\alpha \psi\right)\right]\right)_{L^{2}(\Gamma)} d t \\
& +\mathcal{O}\left(\int_{0}^{T}\left\|\nabla \phi_{t}\right\|_{L^{2}(\Gamma)}^{2} d t+\epsilon \int_{0}^{T} E_{\phi}(t) d t\right)+C_{\epsilon} \text { l.o.t. }\left(\phi, \phi_{t}, \psi\right),
\end{aligned}
$$

after use of the regularity properties of $A_{D}^{-1}$ and the commutator. Now using Théorème 3 of [1] (p. 13), and trace theory, we deduce that $\frac{\partial}{\partial \nu} D \gamma_{0} \in \mathcal{L}\left(H^{1}(\Omega), H^{-\frac{1}{2}}(\Gamma)\right)$. With this information, we have then

$$
\begin{aligned}
& \int_{\tau}^{s}\left(\phi_{t}, \frac{\partial}{\partial \nu}\left[D \gamma_{0}\left(\alpha^{2} \phi_{t}-\alpha \psi\right)\right]\right)_{L^{2}(\Gamma)} \\
= & \mathcal{O}\left(\int_{0}^{T} C_{\epsilon}\left\|\nabla \phi_{t}\right\|_{L^{2}(\Gamma)}^{2} d t+\epsilon \int_{0}^{T}\left[E_{\phi}(t)+\|\nabla \psi(t)\|_{L^{2}(\Omega)}^{2}\right] d t\right)+C_{\epsilon} \text { l.o.t. }\left(\phi, \phi_{t}, \psi\right)(. .5
\end{aligned}
$$


Collecting (33), (34) and (35), we have

$$
\begin{aligned}
& -\int_{\tau}^{s}\left(\Delta \phi_{t t}, A_{D}^{-1}(\alpha \psi)\right)_{L^{2}(\Omega)} d t \\
= & -\int_{\tau}^{s}\left(\phi_{t}, \alpha \psi_{t}\right)_{L^{2}(\Omega)} d t \\
& +\mathcal{O}\left(C_{\epsilon} \int_{0}^{T}\left\|\nabla \phi_{t}\right\|_{L^{2}(\Gamma)}^{2} d t+\epsilon\left\{\left[E_{\phi}(t)\right]_{s}^{\tau}+\int_{0}^{T}\left[E_{\phi}(t)+\|\nabla \psi(t)\|_{L^{2}(\Omega)}^{2}\right] d t\right\}\right) \\
& +C_{\epsilon} \text { l.o.t. }\left(\phi, \phi_{t}, \psi\right) .
\end{aligned}
$$

For the final term in (31), we have by the use of Green's Theorem (6) and the regularity of $A_{D}^{-1}$

$$
\begin{aligned}
& \int_{\tau}^{s}\left(\Delta^{2} \phi-\alpha \psi_{t}+\alpha \operatorname{div}\left[\alpha \nabla \phi_{t}\right]+\nabla \alpha \cdot \nabla \psi+\alpha \psi, A_{D}^{-1}\left(\frac{\alpha}{\gamma} \psi\right)\right)_{L^{2}(\Omega)} d t \\
= & \mathcal{O}\left(\epsilon \int_{0}^{T} E_{\phi}(t) d t\right)+C_{\epsilon} \text { I.o.t. }\left(\phi, \phi_{t}, \psi\right) .
\end{aligned}
$$

The relations (32), (36), and (37), applied to (31), thus give

$$
\begin{aligned}
0= & -\int_{\tau}^{s}\left(\phi_{t}, \alpha \psi_{t}\right)_{L^{2}(\Omega)} d t \\
& +\mathcal{O}\left(C_{\epsilon} \int_{0}^{T}\left\|\nabla \phi_{t}\right\|_{L^{2}(\Gamma)}^{2} d t+\epsilon\left\{\int_{0}^{T}\left[E_{\phi}(t)+\|\nabla \psi(t)\|_{L^{2}(\Omega)}^{2}\right] d t+E_{\phi}(\tau)+E_{\phi}(s)\right\}\right) \\
& +C_{\epsilon} \text { l.o.t. }\left(\phi, \phi_{t}, \psi\right) .
\end{aligned}
$$

Summing (27) and (38), and noting the critical cancellation of the term $\int_{\tau}^{s}\left(\phi_{t}, \alpha \psi_{t}\right)_{L^{2}(\Omega)} d t$, we have

$$
\begin{aligned}
& -\left.\frac{1}{2} E_{\phi}(t)\right|_{t=\tau} ^{t=s}=-\int_{\tau}^{s} \int_{\Omega} \alpha^{2}\left|\nabla \phi_{t}\right|^{2} d \Omega d t \\
& +\mathcal{O}\left(C_{\epsilon} \int_{0}^{T}\left\|\nabla \phi_{t}\right\|_{L^{2}(\Gamma)}^{2} d t+\epsilon\left\{\int_{0}^{T}\left[E_{\phi}(t)+\|\nabla \psi(t)\|_{L^{2}(\Omega)}^{2}\right] d t+E_{\phi}(\tau)+E_{\phi}(s)\right\}\right) \\
& +C_{\epsilon} \text { l.o.t. }\left(\phi, \phi_{t}, \psi\right) \\
\leq & \mathcal{O}\left(C_{\epsilon} \int_{0}^{T}\left\|\nabla \phi_{t}\right\|_{L^{2}(\Gamma)}^{2} d t+\epsilon\left\{\int_{0}^{T}\left[E_{\phi}(t)+\|\nabla \psi(t)\|_{L^{2}(\Omega)}^{2}\right] d t+E_{\phi}(\tau)+E_{\phi}(s)\right\}\right) \\
& +C_{\epsilon} \text { I.o.t. }\left(\phi, \phi_{t}, \psi\right) .
\end{aligned}
$$

This gives Lemma 3.3 (i). The proof of (ii) comes readily from Lemma 3.3 (i), and a rescaling of $\epsilon>0$.

By multiplying the backwards heat equation in (4) by $\psi$, integrating in time and space, and subsequently invoking Green's formula, we have

$$
\begin{aligned}
& \frac{1}{2}\|\psi(0)\|_{L^{2}(\Omega)}^{2}+\int_{0}^{T}\left[\|\nabla \psi\|_{L^{2}(\Omega)}^{2} d t+\|\psi\|_{L^{2}(\Omega)}^{2}+\lambda\|\psi\|_{L^{2}(\Gamma)}^{2}\right] d t \\
= & \int_{0}^{T}\left[\left(\alpha \nabla \phi_{t}, \nabla \psi\right)_{L^{2}(\Omega)}-\left(\alpha \frac{\partial \phi_{t}}{\partial \nu}, \psi\right)_{L^{2}(\Omega)}\right] d t
\end{aligned}
$$


(recall that $\psi(T)=0$ ). Majorizing this expression, in part by the inequality $a b \leq \epsilon a^{2}+C_{\epsilon} b^{2}$ ), we then arrive at the following:

Proposition 3.4 The thermal component of the backwards system (4) satisfies the following inequality:

$$
\int_{0}^{T}\|\nabla \psi\|_{L^{2}(\Omega)}^{2} d t \leq C_{\alpha} \int_{0}^{T}\left[\left\|\nabla \phi_{t}\right\|_{L^{2}(\Gamma)}^{2}+E_{\phi}(t)\right] d t
$$

\section{Proof Proper of Theorem 1.2}

For any $\epsilon \in\left(0, \frac{T}{2}\right)$, we have by two applications of Lemma 3.3(ii)

$$
\begin{aligned}
\int_{0}^{T} E_{\phi}(t) d t= & \int_{0}^{\epsilon} E_{\phi}(t) d t+\int_{T-\epsilon}^{T} E_{\phi}(t) d t+\int_{\epsilon}^{T-\epsilon} E_{\phi}(t) d t \\
\leq & 2 \epsilon E_{\phi}(T)+C \int_{0}^{T}\left\|\nabla \phi_{t}\right\|_{L^{2}(\Gamma)}^{2} d t \\
& +2 \epsilon^{2} \int_{0}^{T}\left[E_{\phi}(t)+\|\nabla \psi\|_{L^{2}(\Omega)}^{2}\right] d t+\int_{\epsilon}^{T-\epsilon} E_{\phi}(t) d t+\text { l.o.t. }\left(\phi, \phi_{t}, \psi\right) \\
\leq & 2 \epsilon E_{\phi}(T)+C_{\epsilon, T} \int_{0}^{T}\left\|\nabla \phi_{t}\right\|_{L^{2}(\Gamma)}^{2} d t+2 \epsilon^{2} \int_{0}^{T}\left[E_{\phi}(t)+\|\nabla \psi\|_{L^{2}(\Omega)}^{2}\right] d t \\
& + \text { l.o.t. }\left(\phi, \phi_{t}, \psi\right),
\end{aligned}
$$

after using Lemma 3.2. Combining this estimate now with that in Proposition 3.4, and subsequently taking $\epsilon>0$ small enough yields

$$
\int_{0}^{T}\left[E_{\phi}(t)+\|\nabla \psi(t)\|_{L^{2}(\Omega)}^{2}\right] d t \leq \epsilon E_{\phi}(T)+C_{\epsilon, T} \int_{0}^{T}\left\|\nabla \phi_{t}\right\|_{L^{2}(\Gamma)}^{2} d t+\text { l.o.t. }\left(\phi, \phi_{t}, \psi\right) .
$$

(Here, we also have implicitly rescaled $\epsilon$, for the sake of clarity only).

To finish the proof, we have by Lemma 3.3(i)

$$
\begin{aligned}
& \int_{0}^{T} E_{\phi}(t) d t=T E_{\phi}(T) \\
& +\mathcal{O}\left(\int_{0}^{T}\left\|\nabla \phi_{t}\right\|_{L^{2}(\Gamma)}^{2} d t+\epsilon\left\{T E_{\phi}(T)+(1+T) \int_{0}^{T} E_{\phi}(t) d t+T \int_{0}^{T}\|\nabla \psi(t)\|_{L^{2}(\Omega)}^{2} d t\right\}\right) \\
& + \text { l.o.t. }\left(\phi, \phi_{t}, \psi\right) .
\end{aligned}
$$

Combining (42) and (43) then yields

$$
\begin{aligned}
& \int_{0}^{T}\left[\frac{1}{2} E_{\phi}(t)+\|\nabla \psi(t)\|_{L^{2}(\Omega)}^{2}\right] d t+\frac{T}{2} E_{\phi}(T) \\
\leq & C_{T} \int_{0}^{T}\left\|\nabla \phi_{t}\right\|_{L^{2}(\Gamma)}^{2} d t+\epsilon(1+T) E_{\phi}(T)+\epsilon(1+T) \int_{0}^{T} E_{\phi}(t) d t \\
& +\epsilon T \int_{0}^{T}\|\nabla \psi(t)\|_{L^{2}(\Omega)}^{2} d t+\text { l.o.t. }\left(\phi, \phi_{t}, \psi\right) .
\end{aligned}
$$


From here, one has the following inequality for $\epsilon>0$ small enough:

$$
\int_{0}^{T}\left[E_{\phi}(t)+\|\nabla \psi(t)\|_{L^{2}(\Omega)}^{2}\right] d t+E_{\phi}(T) \leq C_{T} \int_{0}^{T}\left\|\nabla \phi_{t}\right\|_{L^{2}(\Gamma)}^{2} d t+\text { I.o.t. }\left(\phi, \phi_{t}, \psi\right) .
$$

Note that the preliminary observability inequality above, polluted by lower order terms, reflects no contribution from thermal control. That is to say, there is no term $C_{T}\|\psi\|_{\left[H^{s}(\Sigma)\right]^{\prime}}^{2}$ appearing on the right hand side of (44) (c.f., (7)). In order to eliminate the lower order terms appearing in (44), one must at this point invoke a (by now) classical compactness-uniqueness argument which makes use of the new unique continuation results in [16] (in the case that $\Gamma_{*}=\Gamma$ ) and [6] (in the case that $\Gamma_{*} \subset \Gamma$ ) for the linear system of thermoelasticity (4), in combination with the backwards uniqueness result of [25]; these being used to handle the thermoelastic system with variable coefficient $\alpha$. Namely, If $\left[\varpi, \varpi_{t}, \vartheta\right] \in C\left([0, T] ; H^{2}(\Omega) \times H^{1}(\Omega) \times L^{2}(\Omega)\right)$ solves

$$
\left\{\begin{array}{l}
\left\{\begin{array}{l}
\varpi_{t t}-\gamma \Delta \varpi_{t t}+\Delta^{2} \varpi+\operatorname{div}[\alpha(x, y) \nabla \vartheta]=0 \\
\vartheta_{t}+\Delta \vartheta-\vartheta-\operatorname{div}\left[\alpha(x, y) \nabla \varpi_{t}\right]=0
\end{array} \text { on } Q\right. \\
\left\{\begin{array}{l}
\Delta \varpi+(1-\mu) B_{1} \varpi+\alpha \vartheta=0 \\
\frac{\partial \Delta \varpi}{\partial \nu}+(1-\mu) \frac{\partial B_{2} \varpi}{\partial \tau}-\gamma \frac{\partial \varpi_{t t}}{\partial \nu}+\alpha \frac{\partial \vartheta}{\partial \nu}-\varpi=0
\end{array} \text { on } \Sigma\right. \\
\varpi=\frac{\partial \varpi}{\partial \nu}=0 \text { on } \Sigma \\
\frac{\partial \vartheta}{\partial \nu}+\lambda \vartheta=0 \text { on } \Sigma, \quad \lambda \geq 0 \\
\vartheta=0 \text { on } \Sigma_{*} ;
\end{array}\right.
$$

then for $T>2 \sqrt{\gamma} \operatorname{diam}(\Omega)$, necessarily $\left[\varpi, \varpi_{t}, \vartheta\right]=[0,0,0]$.

It is only here at the compactness-uniqueness level that the thermal trace term appears (In consequence, the parameter $s$ may be allowed any value.) These details of the contradiction argument, being very similiar to those in [3] are omitted. So given the existence of the inequality (44), we obtain by means of compactness-uniqueness the inequality

$$
\text { I.o.t. }\left(\phi, \phi_{t}, \psi\right) \leq C_{T}\left(\int_{0}^{T}\left\|\nabla \phi_{t}\right\|_{L^{2}(\Gamma)}^{2} d t+\|\psi\|_{\left[H^{s}\left(\Sigma_{*}\right)\right]^{\prime}}^{2}\right) \text {. }
$$

Combining (44) and (45) now establishes (7), and so completes the proof of Theorem 1.2.

\section{Appendix}

In the work above, we will have need of the following trace regularity result, proved in [19], for solutions of Kirchoff plates:

Lemma 4.1 Let the function $\phi(t, x)$ satisfy the following Kirchoff equation on an open, bounded domain $\Omega \subset \mathbb{R}^{n}$, with smooth boundary $\Gamma$ :

$$
\left\{\begin{array}{l}
\varphi_{t t}-\gamma \Delta \varphi_{t t}+\Delta^{2} \varphi=f \text { on } Q \\
\left\{\begin{array}{l}
\Delta \varphi+(1-\mu) B_{1} \varphi=g_{1} \\
\frac{\partial \Delta \varphi}{\partial \nu}+(1-\mu) \frac{\partial B_{2} \varphi}{\partial \tau}-\gamma \frac{\partial \varphi_{t t}}{\partial \nu}=g_{2}
\end{array} \text { on } \Sigma\right.
\end{array}\right.
$$


where the boundary operators $B_{i}$ are as in (2). Let $0<\epsilon_{0}<T$ and $\epsilon>0$ be arbitrary. Then one has the inequality

$$
\begin{aligned}
& \int_{\epsilon_{0}}^{T-\epsilon_{0}} {\left[\left\|\frac{\partial^{2} \varphi}{\partial \tau^{2}}\right\|_{L^{2}(\Gamma)}^{2}+\left\|\frac{\partial^{2} \varphi}{\partial \nu \partial \tau}\right\|_{L^{2}(\Gamma)}^{2}+\left\|\frac{\partial^{2} \varphi}{\partial \nu^{2}}\right\|_{L^{2}(\Gamma)}^{2}\right] d t } \\
& \leq C_{T, \epsilon_{0}, \gamma}\left\{\int_{0}^{T}\left[\|f\|_{\left[H^{\frac{3}{2}-\epsilon}(\Omega)\right]^{\prime}}^{2}+\left\|g_{1}\right\|_{L^{2}(\Gamma)}^{2}+\|\varphi\|_{H^{\frac{3}{2}+\epsilon}(\Omega)}^{2}+\left\|\nabla \varphi_{t}\right\|_{L^{2}(\Gamma)}^{2}\right] d t\right. \\
&\left.+\left\|g_{2}\right\|_{H^{-1}(\Sigma)}^{2}\right\} .
\end{aligned}
$$

A version of the $L^{2}(\Omega) \times H^{-1}(\Omega)$ estimates which is needed in this paper (see the proof of Lemma 3.2 ) is different in formulation and statement from those found in the literature, and for this reason is presented below. With $\gamma>0$, we will consider the following:

$$
\gamma z_{t t}-\Delta z-F z=f \text { in } \Omega \times(0, T),
$$

where the term $F z$ corresponds to a first order differential operator in time and space, with sufficiently smooth coefficients. In addition, $f$ is a forcing term which, for our purposes, will be assumed to have the following form:

$$
f=\frac{d}{d t} f_{1}+f_{2}, \text { with } f_{1} \in L^{2}(Q) \cap C\left([0, T] ; H^{-\frac{1}{2}+\epsilon}(\Omega)\right) ; f_{2} \in L^{2}\left(0, T ; H^{-1}(\Omega)\right) .
$$

With $f$ as defined, we then have the following $L^{2}(\Omega) \times H^{-1}(\Omega)$ observability estimate with respect to the wave equation (46).

Theorem 4.2 Let $T>2 \sqrt{\gamma} \operatorname{diam}(\Omega)$. Then the following estimate is valid for all $t \in[0, T]$ :

$$
\begin{aligned}
& \|z(t)\|_{L^{2}(\Omega)}^{2}+\left\|z_{t}(t)\right\|_{H^{-1}(\Omega)}^{2} \\
\leq & C_{T}\left\{\|z\|_{L^{2}(\Sigma)}^{2}+\left\|\frac{\partial z}{\partial \nu}\right\|_{H^{-1}(\Sigma)}^{2}+\|z\|_{H^{-1}(Q)}^{2}\right. \\
& \left.+\int_{0}^{T}\left[\left\|f_{1}\right\|_{L^{2}(\Omega)}^{2}+\left\|f_{2}\right\|_{H^{-1}(\Omega)}^{2}\right] d t+\left\|f_{1}\right\|_{C\left([0, T] ; H^{-\frac{1}{2}+\epsilon}(\Omega)\right)}^{2}\right\} .
\end{aligned}
$$

Proof: With the operator $A_{D}$ as defined in (28), we note by [9] that

$$
\left\|A_{D}^{-\frac{1}{2}} \varpi\right\|_{L^{2}(\Omega)} \sim\|\varpi\|_{H^{-1}(\Omega)} .
$$

Accordingly, we can adopt the following notation for the wave energy at time $t$ :

$$
E_{z}(t)=\frac{1}{2}\|z(t)\|_{L^{2}(\Omega)}^{2}+\frac{\gamma}{2}\left\|A_{D}^{-\frac{1}{2}} z_{t}(t)\right\|_{L^{2}(\Omega)}^{2} .
$$

STEP 1. A Decomposition.

We set $z=w+v$, where

$$
\left\{\begin{array}{l}
\gamma w_{t t}-\Delta w=F w \text { on } Q \\
w=0 \text { on } \Sigma \\
w(0)=z(0) ; w_{t}(0)=z_{t}(0) \text { in } \Omega
\end{array}\right.
$$




$$
\left\{\begin{array}{l}
\gamma v_{t t}-\Delta v=F v+f \text { on } Q \\
v=z \text { on } \Sigma \\
v(0)=0 ; \quad v_{t}(0)=0 \text { in } \Omega
\end{array}\right.
$$

\section{STEP 2. The $w$-Equation.}

For $T>2 \sqrt{\gamma} \operatorname{diam}(\Omega)$, we can directly apply the estimate in Theorem 2 of [30] with $p=-1$, $m=2$. With this result, we then have the existence of an interval $\left[t_{0}, t_{1}\right] \subset[0, T]$ such that

$$
\int_{t_{0}}^{t_{1}} E_{w}(t) d t \leq C_{T}\left(\left\|\frac{\partial w}{\partial \nu}\right\|_{H^{-1}(\Sigma)}^{2}+\|w\|_{H^{-1}(Q)}^{2}\right) .
$$

Next, a classical energy argument with respect to (49) (with multiplier $A_{D}^{-1} w_{t}$ ), an application of Gronwall's inequality, and the inherent time reversibility of the process imply

$$
E_{w}(t) \leq e^{c T} E_{w}(s) \text { for all } s, t \in[0, T]
$$

Therefore,

$$
e^{c T} \int_{t_{0}}^{t_{1}} E_{w}(t) d t \geq E_{w}\left(t_{0}\right)\left(t_{1}-t_{0}\right)
$$

which, with estimate (51), implies

$$
E_{w}\left(t_{0}\right) \leq C_{T}\left(\left\|\frac{\partial w}{\partial \nu}\right\|_{H^{-1}(\Sigma)}^{2}+\|w\|_{H^{-1}(Q)}^{2}\right) .
$$

This and another use of $(52)$ then gives for all $t \in[0, T]$

$$
E_{w}(t)+\int_{0}^{T} E_{w}(t) d t \leq C_{T}\left(\left\|\frac{\partial w}{\partial \nu}\right\|_{H^{-1}(\Sigma)}^{2}+\|w\|_{H^{-1}(Q)}^{2}\right) .
$$

STEP 3. The $v$-Equation.

First off, we note that $v$ satisfies the estimate

$$
\left\|\frac{\partial v}{\partial \nu}\right\|_{H^{-1}(\Sigma)} \leq C_{T}\left[\|z\|_{L^{2}(\Sigma)}+\left\|f_{2}\right\|_{L^{1}\left(0, T ; H^{-1}(\Omega)\right)}+\left\|f_{1}\right\|_{L^{1}\left(0, T ; L^{2}(\Omega)\right)}+\left\|f_{1}\right\|_{C\left([0, T] ; H^{-\frac{1}{2}+\epsilon}(\Omega)\right)}\right]
$$

This statement follows essentially from the regularity theory in [21].

To see this, we decompose $v$ as $v=v_{f_{1}}+v_{\left(f_{2}, z\right)}$, where $v_{\left(f_{2}, z\right)}$ solves the original equation (50), but with $f$ replaced with $f_{2}$ therein.

To handle $v_{\left(f_{2}, z\right)}$, the Theorem 2.3 in [21] immediately applies, and so this yields

$$
\left\|\frac{\partial v_{\left(f_{2}, z\right)}}{\partial \nu}\right\|_{L^{2}(\Sigma)} \leq C_{T}\left(\|z\|_{L^{2}(\Sigma)}+\left\|f_{2}\right\|_{L^{1}\left(0, T ; H^{-1}(\Omega)\right)}\right) .
$$

As for the component $v_{f_{1}}$, we introduce $q=\int_{0}^{t} v_{f_{1}}(s) d s$. The $q$ satisfies the PDE

$$
\begin{aligned}
& \gamma q_{t t}-\Delta q=F q+f_{1}(t)-f_{1}(0) \text { on } Q \\
& q=0 \text { on } \Sigma \\
& q(0)=q_{t}(0)=0 .
\end{aligned}
$$


We further decompose $q$ as $q=q_{1}+q_{2}$, where $q_{1}$ satisfies the equation

$$
\begin{aligned}
& \gamma \frac{d^{2} q_{1}}{d t^{2}}-\Delta q_{1}-F q_{1}=f_{1}(t) \quad \text { on } Q \\
& q_{1}=0 \text { on } \Sigma \\
& q_{1}(0)=\frac{d q_{1}}{d t}(0)=0
\end{aligned}
$$

and $q_{2}$ solves

$$
\begin{aligned}
& \gamma \frac{d^{2} q_{2}}{d t^{2}}-\Delta q_{2}-F q_{2}=-f_{1}(0) \quad \text { on } Q \\
& q_{2}=0 \text { on } \Sigma \\
& q_{2}(0)=\frac{d q_{2}}{d t}(0)=0 .
\end{aligned}
$$

By Theorem 2.1 of [21], we have,

$$
\left\|\frac{\partial q_{1}}{\partial \nu}\right\|_{L^{2}(\Sigma)} \leq C_{T}\left\|f_{1}\right\|_{L^{1}\left(0, T ; L^{2}(\Omega)\right)} .
$$

To handle the component $q_{2}$, we use its explicit representation by means of the underlying Cosine operators $[\mathcal{C}(t), \mathcal{S}(t)]($ see $[7])$ :

$$
q_{2}(t)=-\int_{0}^{t} \mathcal{S}(t-s) f_{1}(0) d s=A_{D}^{-1} \mathcal{C}(t) f_{1}(0)-A_{D}^{-\frac{3}{4}-\frac{\epsilon}{2}}\left(A_{D}^{-\frac{1}{4}+\frac{\epsilon}{2}} f_{1}(0)\right) .
$$

Hence,

$$
\begin{aligned}
\left\|\frac{\partial q_{2}}{\partial \nu}\right\|_{L^{2}(\Sigma)} & \leq\left\|D^{*} A_{D} A_{D}^{-1} \mathcal{C}(t) f_{1}(0)\right\|_{L^{2}(\Sigma)}+\left\|\frac{\partial}{\partial \nu} A_{D}^{-\frac{3}{4}-\frac{\epsilon}{2}}\left(A_{D}^{-\frac{1}{4}+\frac{\epsilon}{2}} f_{1}(0)\right)\right\|_{L^{2}(\Sigma)} \\
& \leq C\left(\left\|f_{1}(0)\right\|_{H^{-1}(\Omega)}+\left\|f_{1}(0)\right\|_{H^{-\frac{1}{2}+\epsilon_{(\Omega)}}}\right),
\end{aligned}
$$

where we have used the "sharp" trace regularity for Cosine Operators (see Lemma 3.0 of [21]), and the regular trace theorem as applied to solutions of elliptic problems. Using (60) and (62) with $q=q_{1}+q_{2}$, we obtain

$$
\left\|\frac{\partial q}{\partial \nu}\right\|_{L(\Sigma)} \leq C_{T}\left(\left\|f_{1}\right\|_{L^{1}\left(0, T ; L^{2}(\Omega)\right)}+\left\|f_{1}(0)\right\|_{H^{-\frac{1}{2}+\epsilon}(\Omega)}\right) .
$$

Going back to the variable $v_{f_{1}}$, (63) gives

$$
\left\|\frac{\partial v_{f_{1}}}{\partial \nu}\right\|_{H^{-1}(\Sigma)}=\left\|\frac{\partial q_{t}}{\partial \nu}\right\|_{H^{-1}(\Sigma)} \leq C_{T}\left(\left\|f_{1}\right\|_{L^{1}\left(0, T ; L^{2}(\Omega)\right)}+\left\|f_{1}(0)\right\|_{H^{-\frac{1}{2}+\epsilon}(\Omega)}\right) .
$$

The estimates (56) and (64) yield now the assertion (55).

In the same way (or instead by using the classical regularity estimates posted in [8],[31],[22]), there is also the estimate

$$
\|v\|_{H^{-1}(Q)} \leq C_{T}\left(\|z\|_{L^{2}(\Sigma)}+\int_{0}^{T}\left[\left\|f_{1}\right\|_{L^{2}(\Omega)}+\left\|f_{2}\right\|_{H^{-1}(\Omega)}\right] d t+\left\|f_{1}\right\|_{C\left([0, T] ; H^{-\frac{1}{2}+\epsilon}(\Omega)\right)}\right) .
$$


We next write the solution $v$ of (50) as $v=v_{f}+v_{z}$, where $v_{f}$ corresponds to the contribution of the forcing term $f$, and $v_{z}$ the contribution of boundary term $\left.z\right|_{\Gamma}$. From the known regularity with respect to the wave operator with Dirichlet boundary data (see Theorem 2.3 of [20]; see also [21]), we have for all $t \in[0, T]$,

$$
E_{v_{z}}(t) \leq C_{T}\|z\|_{L^{2}(\Sigma)} .
$$

We now analyze $v_{f}$, which has zero Dirichlet boundary data, zero initial data, and forcing term $f$. Thus, $v_{f}$ satisfies the equation

$$
\begin{aligned}
& \frac{d^{2}}{d t^{2}} v_{f}+A_{D} v_{f}=F\left(v_{f}\right)+f \\
& v_{f}(0)=\frac{d}{d t} v_{f}(0)=0 .
\end{aligned}
$$

We multiply both sides of equation (67) by $A_{D}^{-1} \frac{d}{d t} v_{f}$, integrate in time and space, and subsequently integrate by parts. This gives:

$$
\begin{aligned}
E_{v_{f}}(t) & \leq C \int_{0}^{t} E_{v_{f}}(s) d s+\left|\int_{0}^{t}\left(f_{1 t}+f_{2}, A_{D}^{-1} \frac{d}{d t} v_{f}\right)_{L^{2}(\Omega)} d s\right| \\
& \leq C\left(\int_{0}^{t} E_{v_{f}}(s) d s+\int_{0}^{t}\left\|A_{D}^{-\frac{1}{2}} f_{2}\right\|_{L^{2}(\Omega)}^{2} d s\right)+\left|\int_{0}^{t}\left(f_{1 t}, A_{D}^{-1} \frac{d}{d t} v_{f}\right)_{L^{2}(\Omega)} d s\right| .
\end{aligned}
$$

We estimate now the last term on the right hand side by using the wave equation (67):

$$
\begin{aligned}
& \int_{0}^{t}\left(f_{1 t}, A_{D}^{-1} \frac{d}{d t} v_{f}\right)_{L^{2}(\Omega)} d t=\left[\left(f_{1}, A_{D}^{-1} \frac{d}{d t} v_{f}\right)_{L^{2}(\Omega)}\right]_{s=0}^{s=t}-\int_{0}^{t}\left(f_{1}, A_{D}^{-1} \frac{d^{2}}{d t^{2}} v_{f}\right)_{L^{2}(\Omega)} d t \\
= & \int_{0}^{t}\left(f_{1}, v_{f}-A_{D}^{-1} F v_{f}-A_{D}^{-1} f_{1 t}-A_{D}^{-1} f_{2}\right)_{L^{2}(\Omega)} d t+\left[\left(A_{D}^{-\frac{1}{2}} f_{1}, A_{D}^{-\frac{1}{2}} \frac{d}{d t} v_{f}\right)_{L^{2}(\Omega)}\right]_{s=0}^{s=t} \\
= & \int_{0}^{t}\left(f_{1}, v_{f}\right)_{L^{2}(\Omega)} d t-\int_{0}^{t}\left(f_{1}, A_{D}^{-1}\left(F v_{f}+f_{2}\right)\right)_{L^{2}(\Omega)} d t \\
& +\left[\left(A_{D}^{-\frac{1}{2}} f_{1}, A_{D}^{-\frac{1}{2}} \frac{d}{d t} v_{f}\right)_{L^{2}(\Omega)}-\frac{1}{2}\left\|A_{D}^{-\frac{1}{2}} f_{1}\right\|_{L^{2}(\Omega)}^{2}\right]_{s=0}^{s=t} \\
\leq & \epsilon\left\|A_{D}^{-\frac{1}{2}} \frac{d}{d t} v_{f}(t)\right\|_{L^{2}(\Omega)}^{2}+C_{\epsilon}\left\|A_{D}^{-\frac{1}{2}} f_{1}\right\|_{L^{\infty}\left(0, T ; L^{2}(\Omega)\right)}^{2} \\
& +C \int_{0}^{t}\left(\left\|f_{1}\right\|_{L^{2}(\Omega)}^{2}+\left\|A_{D}^{-\frac{1}{2}} f_{2}\right\|_{L^{2}(\Omega)}^{2}+\left\|v_{f}\right\|_{L^{2}(\Omega)}^{2}\right) d t .
\end{aligned}
$$

Combining (68) and (69), taking $\epsilon>0$ small enough, and subsequently applying Gronwall's inequality yields for all $t \in[0, T]$

$$
E_{v_{f}}(t) \leq C_{T, \epsilon}\left(\left\|f_{1}\right\|_{L^{\infty}\left(0, T ; H^{-1}(\Omega)\right)}^{2}+\int_{0}^{T}\left[\left\|f_{1}\right\|_{L^{2}(\Omega)}^{2}+\left\|f_{2}\right\|_{H^{-1}(\Omega)}^{2}\right] d t\right) .
$$

From (66) and (70), we then have the estimate

$$
E_{v}(t) \leq C_{T}\left(\|z\|_{L^{2}(\Sigma)}+\left\|f_{1}\right\|_{L^{\infty}\left(0, T ; H^{-1}(\Omega)\right)}^{2}+\int_{0}^{T}\left[\left\|f_{1}\right\|_{L^{2}(\Omega)}^{2}+\left\|f_{2}\right\|_{H^{-1}(\Omega)}^{2}\right] d t\right) .
$$


Recalling now that the solution $z$ of (46) has the decomposition $z=w+v$, the estimates (54) and (71) yield then

$$
\begin{aligned}
E_{z}(t) \leq & C_{T}\left(\|z\|_{L^{2}(\Sigma)}^{2}+\left\|\frac{\partial w}{\partial \nu}\right\|_{H^{-1}(\Sigma)}^{2}+\|w\|_{H^{-1}(Q)}^{2}\right. \\
& \left.+\left\|f_{1}\right\|_{L^{\infty}\left(0, T ; H^{-1}(\Omega)\right)}^{2}+\int_{0}^{T}\left[\left\|f_{1}\right\|_{L^{2}(\Omega)}^{2}+\left\|f_{2}\right\|_{H^{-1}(\Omega)}^{2}\right] d t\right) .
\end{aligned}
$$

To refine this right hand side, we use the decomposition $z=v+w$, and the estimates (55), (65) to finally have

$$
\begin{aligned}
E_{z}(t) \leq & C_{T}\left(\|z\|_{L^{2}(\Sigma)}^{2}+\left\|\frac{\partial z}{\partial \nu}\right\|_{H^{-1}(\Sigma)}^{2}+\|z\|_{H^{-1}(Q)}^{2}\right. \\
& \left.+\left\|f_{1}\right\|_{C\left([0, T] ; H^{-\frac{1}{2}+\epsilon}(\Omega)\right)}^{2}+\int_{0}^{T}\left[\left\|f_{1}\right\|_{L^{2}(\Omega)}^{2}+\left\|f_{2}\right\|_{H^{-1}(\Omega)}^{2}\right] d t\right),
\end{aligned}
$$

which is the desired conclusion.

Remark 4.3 We note that one could, in principle, apply the result of [30] to the original equation (46) for $z$. However, by doing so, we would obtain terms involving the anisotropic norms $|f|_{(0, p)}$, which call for an additional regularity near the boundary in the normal direction, a regularity which may not be available. These terms usually create technical difficulties at the level of application. Another reason why we use the decomposition of $z$ into $v$ and $w$ is due to the boundary term $\left.z\right|_{\Sigma}$. Indeed, while the estimate of Theorem 2 in [30] would provide good control of the energy over $\left[t_{0}, t_{1}\right]$, the energy relation for the nonhomogeneous problem becomes complicated, and the final result would have some additional terms which, again, are undesirable here.

Remark 4.4 Instead of applying the results of [30], one might also try using the $L^{2}(\Omega) \times H^{-1}(\Omega)$ estimates previously obtained for the wave equation in [18]. However, an adaptation of these estimates to the present situation would be more complicated than our current methodology.

Remark 4.5 An interesting observation here is that while the task of obtaining $L^{2}(\Omega) \times H^{-1}(\Omega)$ recovery estimates from basic energy level $H^{1}(\Omega) \times L^{2}(\Omega)$ estimates appears very natural, the actual process involved in doing this is a subtle one. Indeed, the usual way of "shifting down" the estimates by one unit, relies on the multiplication of the equation by a tangential operator of order -1 , and then running the usual (energy level) estimates for the transformed problem. However, in this case, the procedure runs into problems, owing to the appearance of commutator terms which cannot be handled by the estimates. For this reason, the particular choice of norm rescaling tangential operator is very important. In fact, in [18], a special construction of the tangential operator was developed, with the main idea being to keep the commutations close to the elliptic microlocal sector. Subsequently, elliptic regularity was used to absorb the terms resulting from the commutations. In [30], instead, the same goal was achieved by a very clever cutoff function which was able to produce the right sign for the troublesome commutators.

\section{References}

[1] J. P. Aubin, Analyse Fonctionelle Appliquée Tome 2, Presses Universitaire de France (1979). 
[2] G. Avalos and I. Lasiecka, Exponential stability of a thermoelastic system with free boundary conditions without mechanical dissipation, SIAM Journal of Mathematical Analysis, Vol. 29, No. 1 (January 1998), pp. 155-182.

[3] G. Avalos and I. Lasiecka, Boundary controllability of thermoelastic plates with free boundary conditions, IMA Preprint Series \#1567 (June 1998), and to appear in SIAM Journal on Control and Optimization.

[4] N. Carmichael and M. D. Quinn, Fixed point methods in nonlinear control, Proceedings of the Second International Conference, Vorau, Austria, 1984, Springer-Verlag Lecture Notes in Control and Information Sciences, Vol. 75 (1985), pp. 24-51.

[5] M. Eller, Uniqueness of continuation theorems, Proceedings of the ISAAC '97, to appear.

[6] M. Eller, Carleman estimates with a second large parameter, preprint (1999).

[7] H. O. Fattorini, Ordinary differential equations in linear topological spaces, I and II, Journal of Differential Equations, Vol. 5 (1968), pp. 72-105; and Vol. 6 (1969), pp. 537-565.

[8] A. Fursikov and O. Imanuvilov, Controllability of Evolution Equations, Lecture Notes, Seoul National University (1996).

[9] P. Grisvard, Caracterization de quelques espaces d'interpolation, Arch. Rat. Mech. Anal. 25 (1967), pp. 40-63.

[10] S. Hansen and B. Zhang, Boundary control of a linear thermoelastic beam, J. Math. Anal. Appl., 210 (1997), pp. 182-205.

[11] H. Hermes, Controllability and the singular problem, SIAM Journal on Control and Optimization, Vol. 2 (1965), pp. 241-260.

[12] V. Hutson and J. S. Pym, Applications of Functional Analysis and Operator Theory, Academic Press, New York (1980).

[13] O. Imanuvilev and M. Yamamoto, Inverse parabolic problems by Carleman's estimates, to appear in Inverse Problems.

[14] V. Isakov, Inverse Problems for Partial Differential Equations, Springer-Verlag, New York (1997).

[15] V. Isakov, On the uniquenes in a lateral Cauchy problem with multiple characteristics, Journal of Differential Equations, Vol. 97 (1997), pp. 134-147.

[16] V. Isakov, On the uniqueness of the continuation for a thermoelasticity system, to appear in Journal of Differential Equations.

[17] V. Komornik, Exact Controllability and Stabilization, Masson, Paris (1994).

[18] I. Lasiecka and R. Triggiani, Uniform boundary stabilization of the wave equation with Dirichlet and Neumann feedback control without geometric conditions, Applied Mathematics and Optimization, Vol. 25 (1992), pp. 189-224.

[19] I. Lasiecka and R. Triggiani, Sharp trace estimates of solutions to Kirchoff and Euler-Bernoulli equations, Applied Mathematics and Optimization, 28 (1993), pp. 277-306.

[20] I. Lasiecka and R. Triggiani, Recent advances in regularity of second-order hyperbolic mixed problems, and applications, Dynamics Reported, Vol. 3 (C. K. R. T. Jones, U. Kirchgraber and H. O. Walther, Editors), Springer-Verlag, New York (1994), pp. 104-162. 
[21] I. Lasiecka, J. L. Lions and R. Triggiani, Nonhomogeneous boundary value problems for second order hyperbolic operators, J. Math. Pures et Appl., 65 (1986), pp. 149-192.

[22] I. Lasiecka and R. Triggiani, Carleman's estimates and boundary controllability for a system of coupled, nonconservative, second-order hyperbolic equations, Lecture Notes in Pure and Applied Mathematics, Marcel Dekker, Vol. 188 (1997), pp. 215-245.

[23] I. Lasiecka and R. Triggiani, Structural decomposition of thermoelastic semigroups with rotational forces, Semigroup Forum, 5 (1999), pp. 585-599.

[24] I. Lasiecka and R. Triggiani, Sharp regularity theory for elastic and thermo-elastic Kirchoff equations with free boundary conditions, to appear in the Rocky Mountain Journal of Mathematics.

[25] I. Lasiecka, M. Renardy and R. Triggiani, Backward uniqueness property for thermoelastic plates, preprint (1999).

[26] J. Lagnese, Boundary Stabilization of Thin Plates, SIAM Stud. Appl. Math. 10, SIAM, Philadelphia, PA (1989).

[27] J. Lagnese, The reachability problem for thermoelastic plates, Arch. Rational Mech. Anal., 112 (1990), pp. 223-267.

[28] W. Liu, Partial exact controllability and exponential stabilizability in higher dimensional linear thermoelasticity, ESAIM: Control, Optimisation and Calculus of Variations, Vol. 3 (1998), pp. 23-48; and Erratum on Partial exact controllability and exponential stabilizability in higher dimensional linear thermoelasticity, ESAIM: Control, Optimisation and Calculus of Variations, Vol. 3 (1998), pp. 323-328.

[29] D. L. Russell, Controllability and stabilizability theory for linear partial differential equations, recent progress and open questions, SIAM Review 20 (1978), pp. 639-739.

[30] D. Tataru, A priori estimates of Carleman's type in domains with boundary, J. Math. Pures Appl., 73 (1994), pp.355-387.

[31] D. Tataru, Exact controllability of hyperbolic and ultrahyperbolic PDE's, Ph. D. Thesis, University of Virginia (1992). 